\title{
Developmental Abnormalities in Mouse Embryos Tetrasomic for Chromosome 11: Apparent Similarity to Embryos Functionally Disomic for the X Chromosome
}

\author{
Hiromichi Mizuno, Ikuhiro Okamoto and Nobuo Takagi* \\ Division of Bioscicence, Graduate School of Environmental Earth Science, Hokkaido University \\ North 10, West 5, Kita-ku, Sapporo 060-0810, Japan
}

(Received 1 April 2002, accepted 5 July 2002)

\begin{abstract}
Adopting a mating system involving two different Robertsonian translocations with monobrachial homology, we studied the early development of mouse embryos trisomic or tetrasomic for chromosome 11. A developmental delay of 12-24 hours was evident in trisomic embryos at embryonic day (E)7.5, whereas tetrasomic embryos apparently had stopped growth by E6.5 without formation of extraembryonic structures. This extremely severe developmental abnormality found in tetrasomic embryos is similar to that reported in embryos having two active $\mathrm{X}$ chromosomes in extraembryonic cell lineages. Autosomal tetrasomy, but not autosomal trisomy, can lead to such early developmental errors. Thus, a reasonable inference would be that the $\mathrm{X}$ chromosome is twice as active as the autosome. Probably, the $\mathrm{X}$ chromosome became upregulated in response to the evolutionary necessity of minimizing haplo-insufficiency brought about by miniaturization of the Y chromosome.
\end{abstract}

\section{INTRODUCTION}

The availability of mouse stocks having various Robertsonian translocations (Beechey and Evans, 1996) has facilitated systematic studies of the developmental effects of increases or decreases in the copy number of a specific autosome (Gropp et al., 1974, 1975). Embryos trisomic for any autosome survive at least until midgestation, although the stage of lethality varies widely among different chromosomes (Epstein, 1986). Autosomally monosomic embryos, on the other hand, survive at most until immediately after implantation (Baranov, 1983), and all nullisomic embryos studied so far die even earlier, probably at early cleavage stages (Magnuson and Epstein, 1981; Magnuson et al., 1985). Thus, each of these aneusomic groups constitutes a well-defined class with respect to the developmental potential. However, not much is known about tetrasomics. The phenotypic effects of two excess copies of an autosome have been analyzed only for chromosomes 15 and 16. Embryos tetrasomic for chromosome 15 are reported to be minute or "streak" embryos at embryonic day (E)8.5 to 9.5, and obviously would not survive much beyond E9.5 (Beechey and Searle, 1988). Tetrasomics for chromosome 16, on the other hand, live longer than tetrasomy 15 embryos, and are

Edited by Toshihiko Shiroishi

* Corresponding author. E-mail: ntakagi@ees.hokudai.ac.jp dead or dying by E12.5 (Debrot and Epstein, 1986). It is likely that tetrasomic embryos are more severely affected than embryos trisomic for the same chromosome. No histological information has been available about these tetrasomic embryos.

Mouse embryos having an additional $\mathrm{X}$ chromosome from the mother stop growing soon after implantation, primarily because the two maternally inherited $\mathrm{X}$ chromosomes are active in extraembryonic cell lineages due to parental imprinting (Shao and Takagi, 1990; Goto and Takagi, 1998). Growth abnormalities found in these embryos are considerably severer than those reported in embryos trisomic for any autosome. In this study we examined the development of embryos trisomic or tetrasomic for chromosome 11 making use of mice carrying two different Robertsonian translocations with monobrachial homology for chromosome 11 to gain further insight into effects of autosomal tetrasomy in mice. The present findings and circumstantial evidence demonstrate that tetrasomies and trisomies constitute two different groups with partial overlap, and embryos functionally disomic for the $\mathrm{X}$ chromosome are more like autosomal tetrasomics than trisomics. This may be because the $\mathrm{X}$ chromosome carries a greater number of genes involved in early embryogenesis or because X-linked genes are, on average, more active than autosomal genes (Ohno, 1967). Recent genetic as well as genomic data favor the view that $\mathrm{X}$ - 
linked genes had been up-regulated during evolution to avoid haplo-insufficiency in males, which have become practically monosomic for the $\mathrm{X}$ chromosome due to degeneration of the $\mathrm{Y}$ chromosome during evolution.

\section{MATERIALS AND METHODS}

Mice. Female mice homozygous for $\mathrm{Rb}(10.11) 8 \mathrm{Bnr}$ (shortened hereafter to Rb8) (Gropp et al., 1972) were mated with males homozygous for Rb(11.13)6Lub (shortened hereafter to Rb6) (Capanna et al., 1976) or vice versa. F1 mice doubly heterozygous for these translocations (which are thus characterized by monobrachial homology for chromosome 11) were mated to produce embryos trisomic or tetrasomic for chromosome 11 taking advantage of the high frequency of meiotic nondisjunction in such mice (Gropp et al., 1975; White et al., 1972).
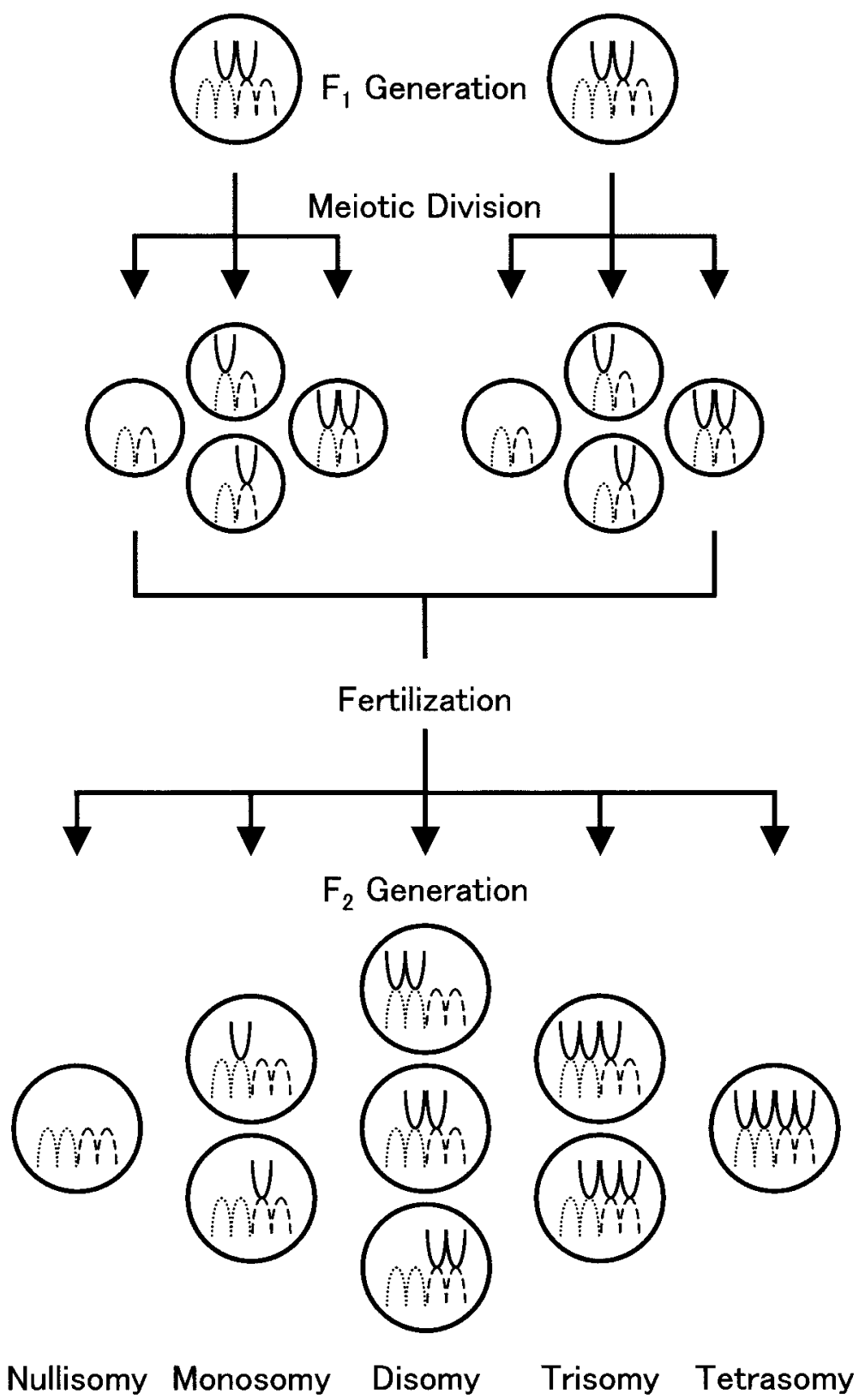

Fig. 1. Production of mouse embryos having 0 to 4 copies of chromosome 11 by mating Rb8 and Rb6 stock mice (Gropp et al., 1972 ; Capanna et al., 1976). F1 mice doubly heterozygous for both translocations (which are thus characterized by monobrachial homology for chromosome 11) were mated to produce embryos trisomic or tetrasomic for chromosome 11 taking advantage of the high frequency of meiotic nondisjunction in such mice (Gropp et al., 1975). Only the three pairs of chromosomes involved in the metacentrics are shown here. Except for a low frequency of non-disjunction involving chromosomes 10 or 13, the remaining 34 acrocentrics segregate normally. 
Table 1. Pre- and post-implantation loss in litters produced by the crosses Rb8/Rb6 $\times$ Rb8/Rb6 and $\mathrm{Rb} 6 / \mathrm{Rb} 8 \times \mathrm{Rb} 6 / \mathrm{Rb} 8$

\begin{tabular}{lcccc}
\hline \hline \multirow{2}{*}{ Stage of embryos } & \multicolumn{4}{c}{ Number of } \\
\cline { 2 - 5 } & Mice copulated & Corpora lutea & Implants & Embryos \\
\hline E6.5 & 9 & & & 60 \\
Rb8/Rb6 $\times$ Rb8/Rb6 & 9 & 87 & 62 & 57 \\
Rb6/Rb8 $\times$ Rb6/Rb8 & 18 & 174 & 124 & 117 \\
Total & & $(9.7)$ & $(6.9)$ & $(6.5)$ \\
& 21 & & & 133 \\
E7.5 & 20 & 203 & 144 & 134 \\
Rb8/Rb6 $\times$ Rb8/Rb6 & 41 & 211 & 144 & 267 \\
Rb6/Rb8 $\times$ Rb6/Rb8 & & 414 & 288 & $(6.5)$ \\
Total & & $(10.1)$ & $(7.0)$ & 384 \\
& 59 & 588 & 412 & $(6.5)$ \\
\hline
\end{tabular}

The mean per litter is given in brackets

Recovery of embryos. Embryos were recovered from pregnant females mostly at E6.5 or 7.5. Noon of the day of detection of a copulation plug was considered E0.5. Recovered embryos were processed either for histology or for karyotype analysis after being photographed. A few pregnant females were sacrificed daily from E8.5 to E12.5 to study the growth of embryos with trisomy 11.

Karyotype analysis. Karyotype analysis was carried out according to the method described earlier (Takagi, 1980). Embryos were incubated at $37^{\circ} \mathrm{C}$ in MEM supplemented with $10 \%$ fetal bovine serum and $150 \mu \mathrm{g} / \mathrm{ml}$ bromodeoxyuridine for $7 \mathrm{hr}$ (E6.5) or $7.5 \mathrm{hr}$ (E7.5), with 0.02 $\mu \mathrm{g} / \mathrm{ml}$ Colcemid added during the last hour. At the end of the incubation, embryos were treated with $1 \%$ sodium citrate and fixed with 3:1 methanol/glacial acetic acid. Chromosome slides were prepared by a routine method and stained with freshly prepared acridine orange. The normal diploid embryo is expected to have 38 chromosomes, including two metacentric chromosomes, in the matings employed here (Fig. 1). If the total chromosome number is 38 , nullisomy 11 is inferred from the complete absence of a metacentric element, and trisomy and tetrasomy 11 are deduced from the presence of three and four metacentric chromosomes, respectively.

Histological analysis. Embryos for histology were embedded in Epon 812 after serial fixation in $3.1 \%$ glutaraldehyde for $2 \mathrm{hr}$ and in $1 \%$ osumium tetraoxide for 30 min. Sections cut at 2- to $3-\mu \mathrm{m}$ thickness were stained with toluidine blue.

\section{RESULTS}

Embryos were recovered at E6.5 or E7.5 from 30 $(\mathrm{Rb} 8 \times \mathrm{Rb} 6) \mathrm{F} 1$ and $29(\mathrm{Rb} 6 \times \mathrm{Rb} 8) \mathrm{F} 1$ females mated with their corresponding males, as outlined in Fig. 1. Data from both crosses were combined, because there were no apparent differences in the average number of ovulated eggs, or pre-implantation or post-implantation loss of
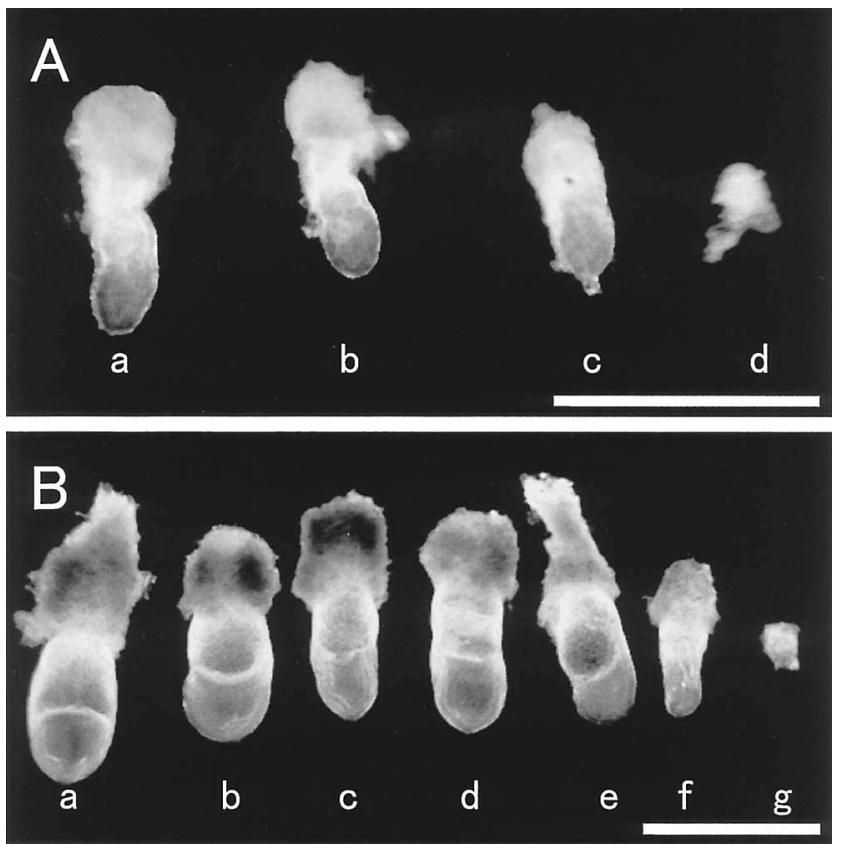

Fig. 2. Gross morphology of mouse embryos recovered at E6.5 (A) and E7.5 (B). Tetrasomy 11 embryos are minute (Ad and $\mathrm{Bg}$ ), whereas trisomy 11 embryos show only slight growth retardation (Ac, Bb-f) in comparison with normal littermates (Aa, b and $\mathrm{Ba}$ ). Scale bar, $1.0 \mathrm{~mm}$. 
embryos. As summarized in Table 1, the average number of ovulated eggs was 10.0, including about 3 lost before implantation. The average number of implantation sites without a living embryo was 0.5 , suggesting that at most one embryo was lost from each female immediately after implantation.

Embryos recovered at E6.5 and E7.5 were divided into three classes based on their gross morphology; normal, delayed and grossly abnormal (Fig. 2). Embryos of the last group were extremely small and morphologically distinct from normal embryos at earlier stages. Karyotype analyses of 199 apparently normal embryos showed that the majority of them had a "normal" balanced karyotype with 38 chromosomes including two metacentric elements (Fig. 3A), whereas eight morphologically normal embryos were trisomic for chromosome 11 with three metacentric and 35 acrocentric chromosomes (Fig. 3B; Table 2). Seven embryos were trisomic for either chromosome 10 or 13. Of 161 retarded embryos 128 had trisomy 11 , whereas 24 embryos were karyotypically balanced. Six retarded embryos were either trisomic for chromosome 10 or 13. Twenty-two embryos were diagnosed as grossly abnormal. Tetrasomy 11 with four metacentric and 34 acrocentric chromosomes (Fig. 3C) was ascertained in 17 of these embryos. Although two abnormal embryos had trisomy 11 and the remaining two were karyotypically balanced, most grossly abnormal embryos at these stages were, thus, considered to be tetrasomic for chromosome 11. Karyotype was not determined for three abnormal embryos. Embryos with tetrasomy 11 were small and almost similar in size at E6.5 and E7.5 suggesting that they had practically stopped growth by E6.5. Embryos
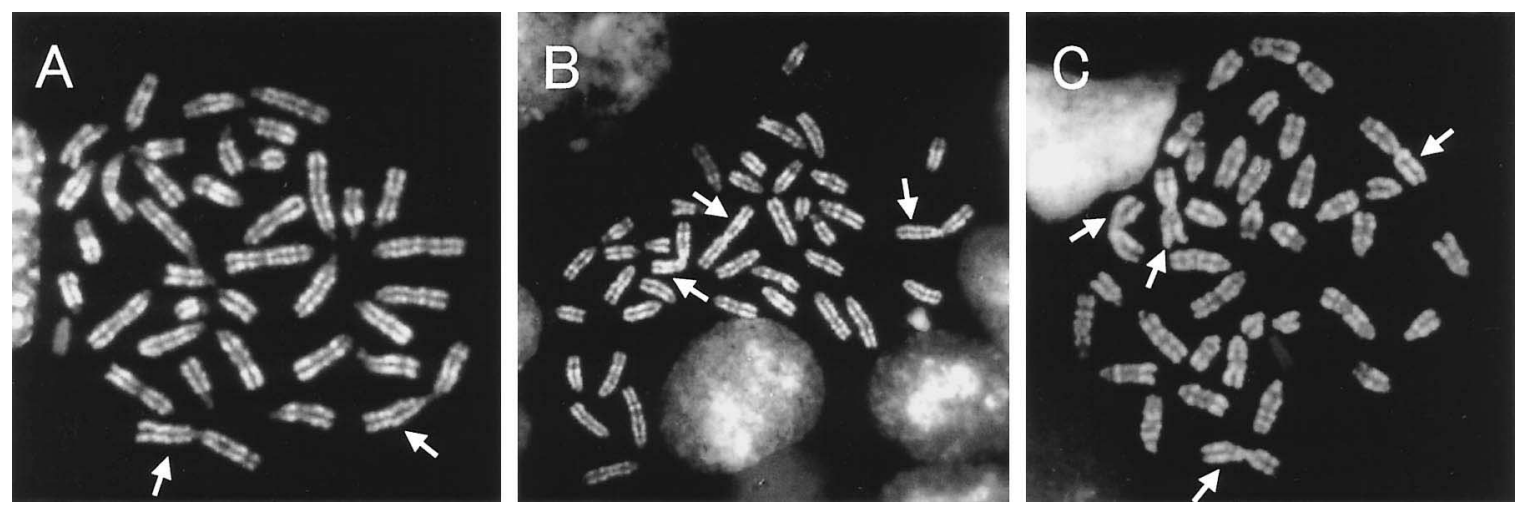

Fig. 3. Representative mitotic metaphase cells of embryos with disomy 11 with 36 acrocentric and 2 metacentric chromosomes (A), trisomy 11 with 35 acrocentric and 3 metacentric chromosomes (B), and tetrasomy 11 with 34 acrocentric and 4 metacentric chromosomes (C). Chromosomes were allowed to replicate in the presence of BrdU during the late $\mathrm{S}$ phase and stained with acridine orange. Arrows indicate metacentric elements involving chromosome 11.

Table 2. Effects of one and two extra copies of chromosome 11 on early development of mouse embryos derived from the crosses Rb8/ $\mathrm{Rb} 6 \times \mathrm{Rb} 8 / \mathrm{Rb} 6$ and $\mathrm{Rb} 6 / \mathrm{Rb} 8 \times \mathrm{Rb} 6 / \mathrm{Rb} 8$

\begin{tabular}{lcccccc}
\hline \hline \multirow{2}{*}{ Growth } & \multicolumn{5}{c}{ Number of embryos } \\
\cline { 2 - 6 } E6.5 & Disomy 11 & Trisomy 11 & Tetrasomy 11 & Others* & N. D.** & Total \\
$\quad$ normal & 54 & 4 & 0 & 3 & 1 & 62 \\
retarded & 5 & 42 & 0 & 0 & 1 & 48 \\
abnormal & 1 & 0 & 6 & 0 & 0 & 7 \\
Total & $60(51.3)^{* * *}$ & $46(39.3)$ & $6(5.1)$ & $3(2.6)$ & $2(1.7)$ & 117 \\
E7.5 & & & & & & 137 \\
normal & 129 & 4 & 0 & 4 & 0 & 113 \\
retarded & 19 & 86 & 0 & 6 & 2 & 17 \\
abnormal & 1 & 2 & 11 & 0 & 3 & 267 \\
Total & $149(55.8)$ & $92(34.5)$ & $11(4.1)$ & $10(3.7)$ & $5(1.9)$ & \\
\hline
\end{tabular}

* Twelve trisomics for other than chromosome 11 and one embryo with 42 chromosomes including three Robertsonian translocations

** Not determined

*** Percentage 
nullisomic or monosomic for chromosome 11 were not found at all after implantation. This is consistent with earlier reports that such hypodiploid embryos are lost before implantation (Beechey and Searle, 1988).

Karyotype analysis was difficult without destroying the integrity of embryos, especially in the case of small and abnormal ones. Consequently, we conducted a histological study on embryos tentatively classified as trisomic and tetrasomic for chromosome 11 based on their gross morphology. As shown in Figs. 4 and 5, putative trisomy 11 embryos showed no specific abnormality except for a developmental delay of 12 to 24 hours. All trisomy 11 embryos consisted of three well-formed primary germ layers, but they could not survive beyond E12.5 (data not shown), in agreement with earlier reports (Gropp et al.,
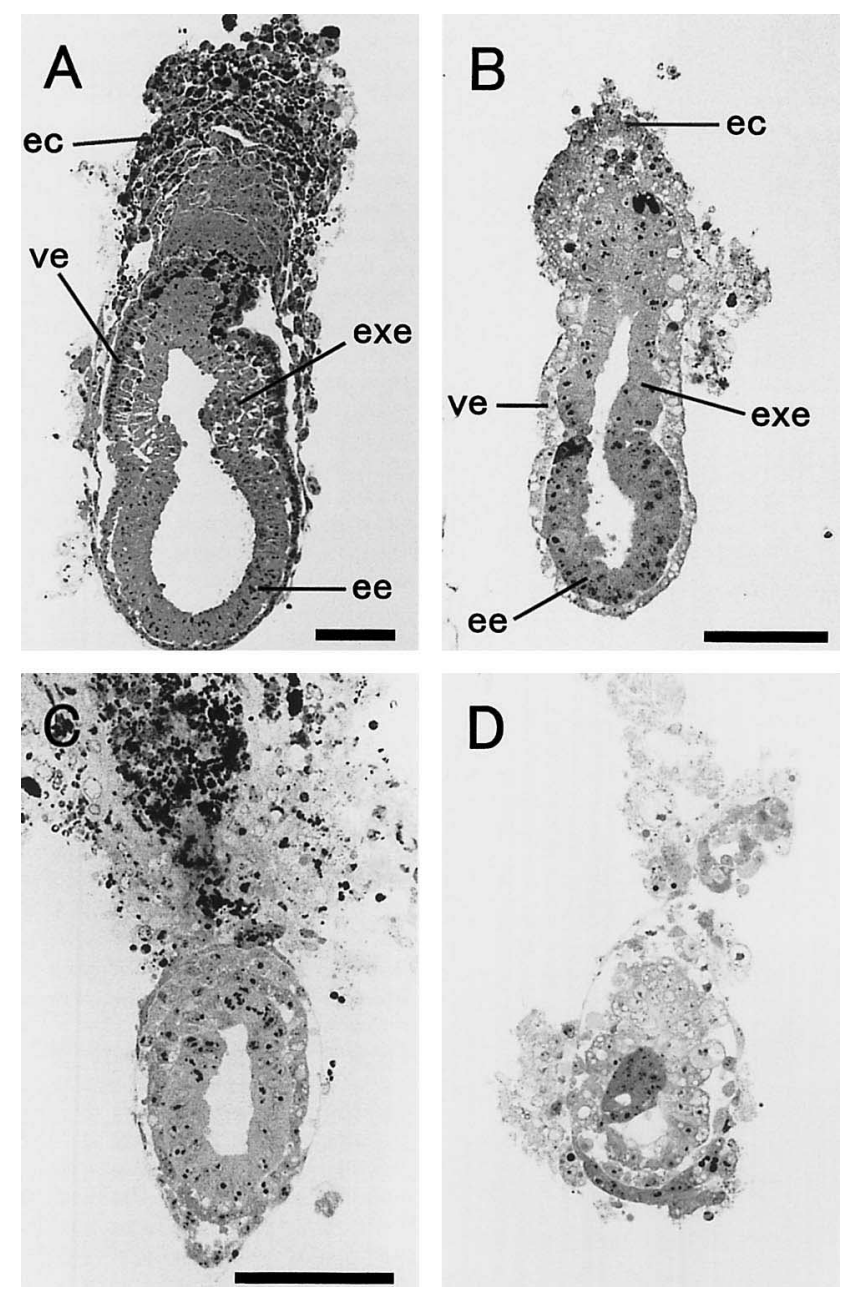

Fig. 4. Histological sections of E6.5 embryos derived from crosses between mice monobrachially homologous for chromosome 11 . A, a normally grown embryo; $\mathrm{B}$, a putative trisomy 11 embryo; $\mathrm{C}$ and $\mathrm{D}$, putative tetrasomy 11 embryos. The trisomy 11 embryo shows a slight developmental delay. Abnormalities in tetrasomy 11 embryos, on the other hand, are severe and show failures of formation of the ectoplacental cone and differentiation into embryonic and extraembryonic regions. Scale bar, $100 \mu \mathrm{m}$. Magnification is identical in C and D.
1974). Abnormalities in presumptive tetrasomy 11 embryos, on the other hand, were severe, and the severity varied slightly from one embryo to the other. The most seriously affected cases were tiny degenerating embryos such as that shown in Fig. 4D. Embryos that grew better consisted of minute embryonic ectoderm vesicles without apparent extraembryonic structures (Figs. 4C, 5D). The development of the E7.5 embryo shown in Fig. 5C is better than that of other presumptive tetrasomy 11 embryos in that it shows the development of extraembryonic structures and the embryonic ectoderm layer. However, both the ectoplacental cone and mesoderm were absent even in this particularly well-developed embryo. Failure of the formation of extraembryonic tissues seems to be mainly responsible for the abnormal embryonic
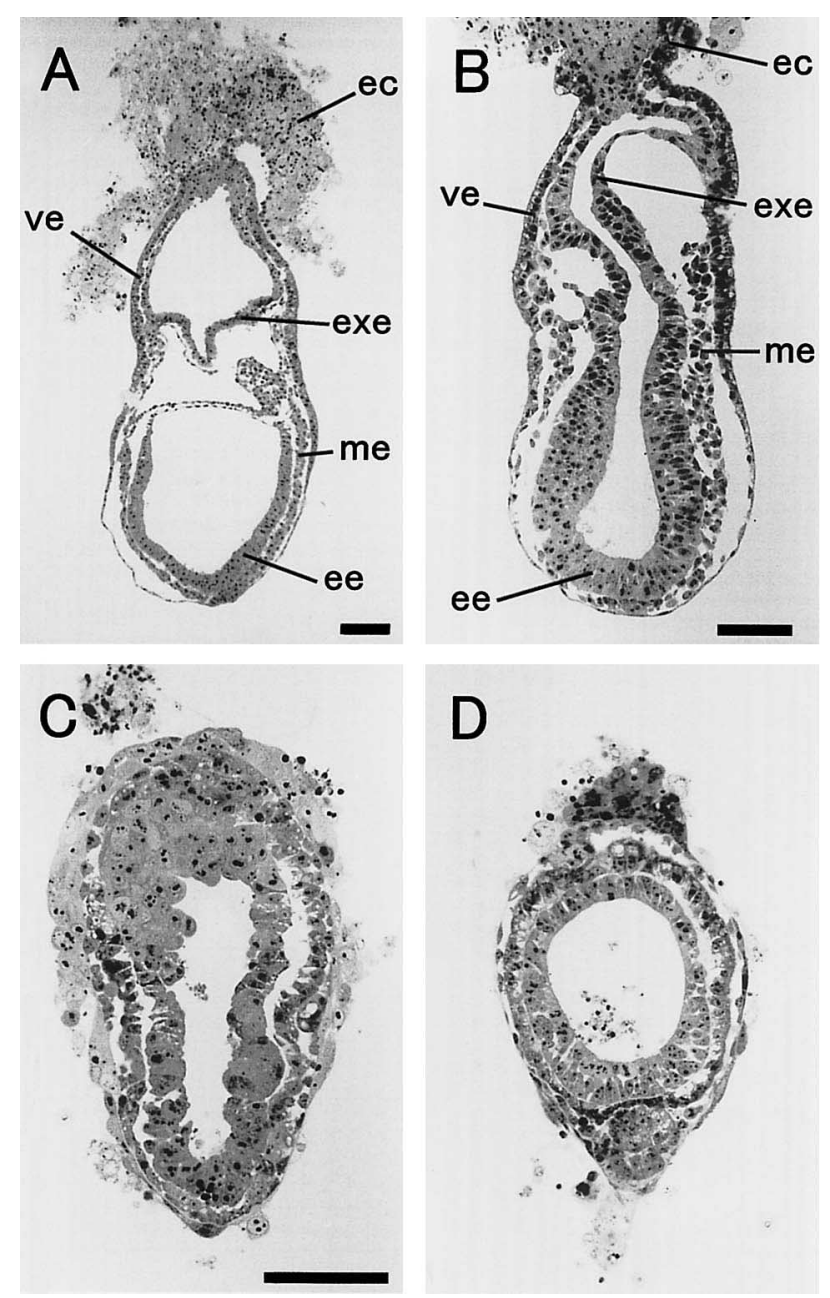

Fig. 5. Histological sections of E7.5 embryos derived from crosses between mice monobrachially homologous for chromosome 11. A, a normally grown embryo; B, a putative trisomy 11 embryo; $\mathrm{C}$ and $\mathrm{D}$, putative tetrasomy 11 embryos. Although the putative tetrasomic embryo shown in $\mathrm{C}$ grew exceptionally well with a distinct extraembryonic region, the one shown in $\mathrm{D}$ is similar in size to those shown in Fig. 4C and 4D), suggesting that tetrasomic embryos stop growth by E6.5. Scale bar, 100 $\mu \mathrm{m}$. Magnification is identical in $\mathrm{C}$ and $\mathrm{D}$. 
growth of these tetrasomics. Thus, the phenotypic effect of two extra copies of chromosome 11 is much more serious than expected from the slight developmental delay of embryos having one extra copy.

The probability (p) of nondisjunction between two Robertsonian translocation chromosomes in $\mathrm{Rb} 6 / \mathrm{Rb} 8$ mice may be calculated from the proportion of embryos tetrasomic for chromosome 11 under two assumptions. First, the probability of nondisjunction is identical in females and males, and second, embryos tetrasomic for chromosome 11 are not lost before E7.5. Based on these assumptions $\mathrm{p}$ was determined to be 0.34 from the correlation: $(\mathrm{p} / 2)^{2}=17 / 588$. The denominator was the total number of ovulated oocytes. The observed frequency of embryos trisomic for chromosome $11(138 / 588=0.235)$ agreed closely with the frequency of 0.229 calculated from $\mathrm{p}(1-\mathrm{p})$.

\section{DISCUSSION}

Autosomal tetrasomies vs. trisomies. Reviewing the then-available cases in humans and mice, Epstein (1986) concluded that while the effects may sometimes be minimal, abnormality increases as the number of times a specific chromosome or segment is represented in the genome is increased. All the mice with full trisomies survive until at least midgestation. The time of their death appears to cluster in three periods: at about E9-11 (chromosomes 2, 3, 5, 7-9, 11, 15, 17), E13-15 (chromosomes 1, 4, 6, 10, 12), and E16 to term (chromosomes 13, 14, 16, $18,19)$, although there is considerable variability in the time of death of individual embryos (Gropp et al., 1974, 1975, 1983; Epstein, 1986; Hernandez and Fisher, 1999)). Our observations showed that trisomy 11 caused a developmental delay of 12-24 hours without any apparent abnormality at E7.5. One more copy of chromosome 11 , however, drastically disturbed early embryonic development culminating in the formation of a tiny embryonic ectoderm vesicle. Conceptuses tetrasomic for chromosome 15 that have been reported to be tiny or "streak" embryos (Beechey and Searle, 1988) may be similar to embryos with tetrasomy 11 . The developmental effects of tetrasomy 16 are less serious than those of tetrasomy 11 or 15 . This may be causally related to the fact that some trisomy 16 mice survive beyond parturition (Miyabara et al., 1982; Haydar et al., 1996), whereas death occurs much earlier in embryos trisomic for chromosome 11 or 15 . It may be postulated that the heavier the damage caused by trisomy of a certain autosome, the severer the phenotye of its tetrasomy. If this is the case, chromosomes 11 and 16 represent two extreme groups of chromosome whose tetrasomy affect mice most and least heavily, respectively. As suggested by Epstein (1986), tetrasomy affects embryonic development considerably more than the corresponding trisomy in mice.
Extraembryonic tissues and $X$ chromosome inactivation in mice. One of the most important tasks of early mammalian embryogenesis is the formation of extraembryonic tissues required for the acquisition of nutrition from the mother. Less than 20 per cent of cells comprising E4.5 blastocysts give rise to the entire fetus, and most of the remaining cells are devoted to the construction of extraembryonic structures. The formation of these extraembryonic structures is affected severely in embryos with tetrasomy 11 . The full development of the extraembryonic tissues is crucial to early embryos: without them, they can not obtain enough nutrients from the mother after implantation, and above all they can not grow normally because the interaction between embryonic and extraembryonic tissues is indispensable for early morphogenesis (Lu et al., 2001). The gross morphology of tetrasomy 11 embryos is reminiscent of embryos that received an extra copy of $\mathrm{X}$ chromosome from the mother (Shao and Takagi, 1990; Goto and Takagi, 1998), and those carrying a paternally transmitted mutant Xist (Marahrens et al., 1997), which is essential for the initiation of X inactivation (Penny et al., 1996), or a maternally transmitted targeted Tsix gene (Lee, 2000; Sado et al., 2001), a probable switch gene of Xist (Lee and Lu, 1999). Extraembryonic tissues are mostly lost in all of these cases and embryos stop growing by E.6.5. X-inactivation does not occur in those nonepiblastic extraembryonic cells in which the future inactive $\mathrm{X}$ chromosome is normally determined by the parental imprinting imposed on the $\mathrm{X}$ chromosome. Functional disomy $\mathrm{X}$ in nonepiblast cells is thus responsible for the loss of extraembryonic structures. Apparent similarities between tetrasomy 11 and functional disomy $\mathrm{X}$ may be fortuitous, but the observations noted above indicate that the developmental effects of one copy of the $\mathrm{X}$ chromosome are nearly equivalent to those of two copies of chromosome 11.

Evolution of sex chromosomes and upregulation of $\mathrm{X}$-linked genes. It has been postulated that the deterioration of the $\mathrm{Y}$ chromosome was initiated when the male-determining $\mathrm{Y}$ chromosome had diverged from the $\mathrm{X}$ chromosome in the ancestors of mammals (Ohno, 1967). Lahn and Page (1999) proposed that inversions that occurred repeatedly in the $\mathrm{Y}$ chromosome were responsible for the inhibition of recombination between $\mathrm{X}$ and $\mathrm{Y}$, and hence the accumulation of mutations in the $\mathrm{Y}$ and its miniaturization. An obvious possibility would be that the X-linked genes forced to become hemizygous by $\mathrm{Y}$ chromosome attrition had been upregulated during evolution to avoid haplo-insufficiency in males (Ohno, 1967). This might have been responsible for the origin of $\mathrm{X}$ chromosome inactivation in females to avoid twice the level of $\mathrm{X}$-linked gene products in each cell, and for the severe developmental abnormalities caused by functional disomy X. In agreement with this view, Adler et al. (1997) 
reported that the activity level of the $\mathrm{Clc4}$ gene, X-linked in Mus spretus but autosomal in laboratory mice, is roughly twice as high in Mus spretus as in Mus musculus.

Systematic analysis of transcripts from the extraembryonic region of E7.5 mouse embryos has recently been reported (Ko et al., 1998). A total of 155 novel cDNA clones were genetically mapped to mouse chromosomes. The distribution of these clones is not uniform among different autosomes and the percentage of mapped genes varies from a high of 9.0 in chromosomes 2 and 17 to a low of 1.9 in chromosomes 12 and 14 . The average percentage for nine chromosomes whose trisomy results in early embryonic death by E11.5 (chromosomes 2, 3, 5, 7$9,11,15,17)$ is 6.02 , whereas that of five chromosomes whose trisomy affects fetal development least heavily (chromosomes 12, 13, 14, 16, 18, 19) is 3.48 . The values are 5.8 for chromosome 11, 4.5 for chromosome 15 and 3.9 for chromosome 16. Although it is necessary to take both genes expressed in embryonic tissues and those expressed later in embryogenesis into account, these findings may explain the differential survival of embryos trisomic for different autosomes. The $\mathrm{X}$ chromosome, in contrast, has the notably low value of 1.3 , although the number of X-linked coding loci make up $4.7 \%$ of all mapped coding loci in mice (Ko et al., 1998). This value is in reasonably good accord with the physical length of the $\mathrm{X}$ chromosome. Because embryos having two active $\mathrm{X}$ chromosomes die immediately after implantation, it is possible that this chromosome carries a small number of highly dosage-sensitive genes that are active at early stages of development. Alternatively, the X chromosome has a unique character that is not yet fully understood. Interestingly, the gene density is lower in the $\mathrm{X}$ chromosome than in any autosome in man (Intern. Hum. Genome Sequencing Consortium, 2001). Further study of the nature of the mammalian $\mathrm{X}$ chromosome is urgently needed. At the same time, studies of embryos tetrasomic for chromosomes other than 11, 15 and 16 will help to clarify the problem.

\section{REFERENCES}

Adler, D. A., Rugarli, E. I., Lingenfelter, P. A., Tsuchiya, K., Poslinski, D., Liggitt, H. D., Chapman, V. M., Elliott, R. W., Ballabio, A. and Disteche, C. M. (1997) Evidence of evolutionary upregulation of the single active $\mathrm{X}$ chromosome in mammals based on Clc4 expression levels in Mus spretus and Mus musculus. Proc. Natl. Acad. Sci. USA 94, 92449248.

Baranov, V. S. (1983) Chromosomal control of early embryonic development in mice I. Experiments on embryos with autosomal monosomy. Genetica 61, 165-177.

Beechey, C. V. and Evans, E. P. (1996) Chromosomal variants. In: Genetic Variants and Strains of the Laboratory Mouse, (eds.: Lyon, M. F., Rastan S. and Brown S. D. M.), pp. 14521506 Oxford Univ. Press, Oxford.

Beechey, C. V. and Searle, A. G. (1988) Effects of zero to four copies of chromosome 15 on mouse embryonic development. Cytogenet. Cell Genet. 47, 66-71.

Capanna, E., Gropp, A., Winking, H., Noack, G. and Civitelli, M. V. (1976) Robertsonian metacentrics in the mouse. Chromosoma 58, 341-353.

Debrot, S. and Epstein, C. J. (1986) Tetrasomy 16 in the mouse: a more severe condition than the corresponding trisomy. J. Embryol. exp. Morph. 91, 169-180.

Epstein, C. J. (1986) The Consequences of Chromosome Imbalance. Principles, Mechanisms, and Models. Cambridge Univ. Press, New York and Cambridge

Epstein, C. J. (1988) Mechanisms of the effects of aneuploidy in mammals. Annu. Rev. Genet. 22, 51-75.

Goto, Y. and Takagi, N. (1998) Tetraploid embryos rescue embryonic lethality caused by an additional maternally inherited X chromosome in the mouse. Development 125, 3353-3363.

Gropp, A., Giers, D. and Kolbus, U. (1974) Trisomy in the fetal backcross progeny of male and female metacentric heterozygotes of the mouse. I. Cytogenet. Cell Genet. 13, 511-535.

Gropp, A., Kolbus, U. and Giers, D. (1975) Systematic approach to the study of trisomy in the mouse. II. Cytogenet. Cell Genet. 14, 42-62.

Gropp, A. and Winking, H. (1981) Robertsonian translocations: cytology, meiosis, segregation patterns and biological consequences of heterozygosity. Zool. Soc. London Symp. 47, 141-181.

Gropp, A., Winking, H., Zech, L. and Muller, H. (1972) Robertsonian chromosomal variation and identification of metacentric chromosomes in feral mice. Chromosoma 39, 265288.

Gropp, A., Winking, H., Herbst, E. W. and Claussen, C. P. (1983). Murine trisomy: developmental profiles of the embryo, and isolation of trisomic cellular systems. J. Exp. Zool. 228, 253-269.

Haydar, T. F., Blue, M. E., Molliver, M. E., Krueger, B. K. and Yarowsky, P. J. (1996). Consequences of trisomy 16 for mouse brain development: corticogenesis in a model of Down syndrome. J. Neurosci. 16, 6175-6182.

Hernandez, D. and Fisher, E. M. C. (1999). Mouse autosomal trisomy: two's company, three's a crowd. Trends in Genetics. 15, 241-247.

Ko, M. S., Threat, T. A., Wang. X., Horton, J. H., Cui, Y., Wang, X., Pryor, E., Paris, J., Wells-Smith, J., Kichen, J. R., Rowe, L. B., Eppig, J., Satoh, T., Brant, L., Fujiwara, H., Yotsumoto, S. and Nakashima, H. (1998) Genome-wide mapping of unselected transcripts from extraembryonic tissue of 7.5day mouse embryos reveals enrichment in the $t$-complex and underrepresentation on the X chromosome. Hum. Mol. Genet. 7, 1967-1978.

Lahn, B. T. and Page, D. C. (1999) Four evolutionary strata on the human X chromosome. Science 286, 964-967.

Lee, J. T. (2000) Disruption of imprinted X inactivation by parent-of-origin effects at Tsix. Cell 103, 17-27.

Lee, J. T. and Lu, N. (1999) Targeted mutagenesis of Tsix leads to nonrandom $\mathrm{X}$ inactivation. Cell 99, 47-57.

Lu, C. C., Brennan, J. and Robertson, E. J. (2001) From fertilization to gastrulation: axis formation in the mouse embryo. Curr. Opin. Genet. Dev. 11, 384-392.

Magnuson, T., Debrot, S., Dimpfl, J., Zweig, A., Zamora, T. and Epstein, C. J. (1985) The early lethality of autosomal monosomy in the mouse. J. Exp. Zool. 236, 353-360.

Magnuson, T. and Epstein, C. J. (1981) Genetic control of very early mammalian development. Biol. Rev. 56, 369-408.

Marahrens, Y., Panning, B., Dausman, J. Strauss, W. and Jae- 
nisch, R. (1997) Xist-deficient mice are defective in dosage compensation but not spermatogenesis. Genes Dev. 11, 156-166.

Miyabara, S., Gropp, A. and Winking, H. (1982) Trisomy 16 in the mouse fetus associated with generalized edema and cardiovascular and urinary tract anomalies. Teratology 25, $369-380$.

Ohno, S. (1967) Sex Chromosomes and Sex Linked Genes. Springer Verlag, Berlin

Penny, G. D., Kay, G. F., Sheardown, S. A., Rastan, S. and Brockdorff, N. (1996) Requirement for Xist in X chromosome inactivation. Nature 379, 131-137.

Sado, T., Wang, Z., Sasaki, H. and Li, E. (2001) Regulation of imprinted X-chromosome inactivation in mice by Tsix. Development 128, 1275-1286.
Shao, C. and Takagi, N. (1990) An extra maternally derived X chromosome is deleterious to early mouse development. Development 110, 969-975.

Takagi, N. (1980) Primary and secondary nonrandom X chromosome inactivation in early female mouse embryos carrying Searle's translocation T (X;16) 16H. Chromosoma 81, 439459.

The International Human Genome Sequencing Consortium (2001) Initial sequencing and analysis of the human genome. Nature 409, 860-921.

White B. J., Tjio, J.-H., Van De Water, L. C. and Crandall, C. (1972) Trisomy 19 in the laboratory mouse I. Frequency in different crosses at specific developmental stages and relationship of trisomy to cleft palate. Cytogenet. Cell Genet. 13, 217-231. 